\title{
Germanica
}

\section{Die Rezeption des Surrealismus in der DDR: die Anthologie Surrealismus in Paris}

La réception du surréalisme en $R D A$ : l'anthologie Surrealismus in Paris

The East German Reception of Surrealism: the Anthology Surrealismus in Paris

Thomas Magnan

\section{CpenEdition}

\section{Journals}

Édition électronique

URL : http://journals.openedition.org/germanica/3505

DOI : 10.4000/germanica.3505

ISSN : 2107-0784

Éditeur

Université de Lille

Édition imprimée

Date de publication : 30 décembre 2016

Pagination : 143-148

ISBN : 9782913857384

ISSN : 0984-2632

\section{Référence électronique}

Thomas Magnan, „Die Rezeption des Surrealismus in der DDR: die Anthologie Surrealismus in Paris“ Germanica [Online], 59 | 2016, Online erschienen am: 30 Dezember 2018, abgerufen am 06 Januar 2021. URL: http://journals.openedition.org/germanica/3505 ; DOl: https://doi.org/10.4000/germanica. 3505

(c) Tous droits réservés 


\title{
Die Rezeption des Surrealismus in der DDR: die Anthologie Surrealismus in Paris
}

\author{
Thomas MAGNAN \\ Université Paris-Sorbonne
}

Die 1986 in der Universal Bibliothek bei Reclam erschienene Anthologie zum Surrealismus ${ }^{1}$ stellt einen wichtigen Meilenstein in der Rezeption der „Moderne“ in der DDR dar, insofern als diese künstlerische Bewegung zum ersten Mal repräsentativ veröffentlicht wird. Nach einer Vorstellung des Werkes und seines Inhalts werden wir auf die problematische Rezeption des Surrealismus und auf die Debatten über die Avantgarde eingehen. Schließlich werden wir die Herausgabe des Buchs und den Druckgenehmigungsvorgang nachzeichnen, anhand des Gutachtens, das im Bundesarchiv einzusehen ist.

Die Anthologie bezieht sich von vornherein auf Paris, die Stadt, die als Geburtsort des Surrealismus gilt: Damit wird die französische Spezifität dieser Gruppe unterstrichen ${ }^{2}$. Die meisten Texte gehören dem begrenzten Zeitraum 1919-1939 an, der den historischen Kern der Bewegung darstellt. Das Buch wurde laut der Druckgenehmigungsakte ${ }^{3}$

1. - Surrealismus in Paris, 1919-1939. Ein Lesebuch, hrsg. von Karlheinz Barck, Leipzig, Reclam Verlag, 1986.

2. - Vgl. Danielle Risterucci-Roudnicky, France-RDA, anatomie d'un transert littéraire, 1949-1990, Bern, Peter Lang, 1999, S. 388.

3. - Druckgenehmigungsakte von Surrealismus in Paris, BArch, DR 1/2218a. 
in 30000 Exemplaren gedruckt, eine hohe Anzahl in Anbetracht der schwierigen, heiklen Rezeption der avantgardistischen Werke in der DDR. Ein Zeichen dafür, dass es die Nachfrage des Publikums traf, ist die neue, veränderte Auflage von 1990.

In der Anthologie werden insgesamt dreißig Künstler dargestellt, die der surrealistischen Bewegung angehörten. Es ist auch bemerkenswert, dass dem Leser nicht nur Literatur dargeboten wird, sondern auch der Beitrag der Surrealisten zum Film und zur Malerei, wobei sechzehn farbige Gemälde abgedruckt sind. Die Texte werden nicht chronologisch geordnet, sondern nach Themen eingeteilt. Der Anfang handelt vom Ursprung des Surrealismus, z.B. mit den Magnetischen Feldern von Breton und Soupault oder dem Ersten Manifest des Surrealismus. Daran anschließend folgen „Traumerzählungen“ und „Surrealistische Texte“. Es gibt auch „Wortspiele“ von u.a. Breton, Duchamp und Desnos und eine große Auswahl von Gedichten, in denen Char, Aragon und Eluard stark repräsentiert sind - es gibt aber nur ein Gedicht von Tristan Tzara. Ein nicht unwichtiger Anteil der Texte ist dem Thema Liebe und Erotik, „Amour fou und Wahn-Sinn“, gewidmet. Hervorzuheben sind auch Texte von Autoren wie Adorno und Peter Weiss, die den Surrealismus aus einer retrospektiven bzw. reflexiven Sicht betrachten, und die den Band schließen.

Die Rezeption surrealistischer Werke findet im Kontext der modernen Literatur in der DDR statt. Bis in die 1970er Jahre bestand eine starke Hierarchie der Literatur, in der der von Lukács theoretisierte Realismus bevorzugt wurde. Den modernen Autoren wie z.B. Kafka, Joyce oder Proust wurde die Kluft zwischen dem Künstler und seiner Umgebung vorgeworfen, sowie die Tendenz zu einer autonomen Kunst. Das kann man z.B. daran sehen, dass die Wortspiele - ein beliebtes literarisches Verfahren der Surrealisten - stark kritisiert und oft nur als Spiel betrachtet wurden.

Vor dem Erscheinen der Anthologie wurden wenige surrealistische Texte veröffentlicht. Der große Vorgänger, auf den sich die Surrealisten beziehen, Lautréamont, wurde dem ostdeutschen Leser nur in Anthologien dargeboten. Auszüge aus seinen Texten kann man in Der Untergang der Romantischen Sonne. Ästhetische Texte von Baudelaire bis Mallarmé (Kiepenheuer, 1. Aufl. 1980, hrsg. von Manfred Starke) lesen. Eine andere Anthologie hatte zuvor einige Texte von Surrealisten vorgestellt: Breton, Soupault, Artaud und Eluard konnte man in Französische Lyrik von Baudelaire bis zur Gegenwart (Reclam 1967, hrsg. von Kurt Schnelle) lesen, es war aber eine Ausnahme.

1985 erschien die erste Ausgabe von Nadja (Insel-Verlag) und damit der erste vollständige Text von Breton in der DDR. Im Verlagsgutachten 
von Brigitte Burmeister ${ }^{4}$ wird die Anthologie des Surrealismus erwähnt, die zu dieser Zeit bei Reclam schon in Vorbereitung war. B. Burmeister hebt die Bedeutung des Nachworts für das Verständnis des Textes hervor, weist aber auch auf eine mangelnde Thematisierung der Avantgarde hin. Sie erwähnt zwei wichtige Werke, die in den 1970er Jahren eine neue Orientierung in der Debatte über die Avantgarde gegeben haben: Künstlerische Avantgarde. Annäherungen an ein unabgeschlossenes Kapitel (1979), herausgegeben von Karlheinz Barck, Dieter Schlenstedt und Wolfgang Thierse; und Theorie der Avantgarde von Peter Bürger (1974). Für B. Burmeister sollte das Nachwort zu Nadja die wesentliche Unterscheidung der Avantgarde zu einer ,bürgerlichen“ Moderne besser darstellen.

Die Anthologie des Surrealismus öffnet auch den Weg zu weiteren Publikationen, wiez.B. dem 1989 erschienenen Gedichtband von Soupault Bitte schweigt (Kiepenheuer). Im Gutachten für die Druckgenehmigung des Buchs ${ }^{5}$ rechtfertigt Helmut Melzer die Veröffentlichung, indem er von einer ,aktuellen kulturpolitischen Situation“ spricht. Er bezieht diese neue Situation auf ein neues Verständnis der Avantgarde.

Daraus ergibt sich, dass die von Karlheinz Barck herausgegebene Anthologie in einem günstigen Kontext in der DDR erscheint. Sie ist in die Debatten der Zeit eingeschrieben: „l'anthologie [...] est l'émergence pour grand public des débats théoriques sur l'avant-garde ${ }^{6}$." Die Anthologie erscheint somit als das Ergebnis einer langwierigen Arbeit von Karlheinz Barck über die Avantgarde. Danielle RisterucciRoudnicky beschreibt die neue Auffassung, die von Barck vertreten wird, folgendermaßen: ,[L'avant-garde] exprime, de façon radicale, la crise de la fonction de l'art bourgeois traditionnel, de la perte de son évidence, la recherche d'autres fonctions. Changer l'art et changer la vie, ces deux buts sont indissociables dans l'art d'avant-garde 7." Es wird hier versucht, eine Verbindung zum Sozialismus herzustellen, insofern als die Kunst von den anderen Bereichen des Lebens nicht getrennt zu sein scheint. Hinzu kommt eine antibürgerliche Kraft. D. RisterucciRoudnicky unterstreicht auch die besondere Rolle von Anthologien. Es handelt sich um ein ,privilegiertes Mittel des kulturellen Transfers8“, eine Form, die es ermöglicht, eine Art „subversive“ Rolle zu haben, indem Texte leichter publiziert werden können, die sonst nie erscheinen würden ${ }^{9}$.

\footnotetext{
4. - Verlagsgutachten von Nadja, BArch, DR1/3484.

5. - Gutachten von Bitte schweigt: BArch, DR1/3705.

6. - D. Risterucci-Roudnicky, France-RDA, a.a.O., S. 381.

7. - Ebd., S. 386.

8. - „un moyen privilégié de transfert culturel“, ebd., S. 155.

9. - Vgl. ebd., S. 162.
} 
Die Herausgabe der Anthologie lässt sich anhand des Druckgenehmigungsvorgangs und des Nachworts nachvollziehen. Die Rolle des Nachworts liegt nämlich darin, den Leser auf die Lektüre der Texte vorzubereiten und ihm die Schlüssel zu einer angemessenen Interpretation zu geben ${ }^{10}$. In seinem Nachwort denunziert Karlheinz Barck, dass der Surrealismus im Westen zu einem Teil der Kulturindustrie geworden ist, vom Kapitalismus integriert und als Konsumprodukt manipuliert, was den Surrealismus von seinem ursprünglichen Zweck weit entfernt: „Als Kunst sind seine Resultate in die Museen und Handbücher eingegangen. Seine provokativen Techniken gehören im Westen längst zum Repertoire der Bedürfnismanipulation 11." Hingegen betont Barck den Bezug des Surrealismus zur Revolution. Er nennt einen „Bezug zu 1789“ und spricht von einer „Revolutionserwartung“ und von „radikalen antibürgerlichen Kräften“, auf denen die Bewegung beruhe. „Die Surrealisten trafen den Nerv der kritischen intellektuellen Nachkriegsgeneration vor allem aber durch den moralischen und bewußt provokatorisch vorgetragenen Anspruch, Poesie und Revolution miteinander zu verbinden ${ }^{12}$." Diese Politisierung der Bewegung begründet für Barck den Unterschied zwischen Avantgarde und Moderne ${ }^{13}$.

Dieser letzte Punkt kann auch als Strategie betrachtet werden, um die Publikation des Buchs zu sichern. Barck unterstreicht die Vielfalt und Widersprüchlichkeit des Surrealismus. Er sorgt dafür, die problematischen bzw. polemischen Aspekte des Surrealismus zu entschärfen. Man kann eine doppelte Bewegung von Aneignung (Bezug zur Revolution) und Kritik feststellen. So verweist er z.B. auf den starken männlichen Chauvinismus der Surrealisten, er denunziert „die phallokratische Erotik der Surrealisten“, zeigt, wie die Surrealisten die Frau auf ihre Rolle als Sexualobjekt oder als Muse reduzieren. Die automatische Schreibweise bedarf auch einer Erklärung, um nicht sinnlos zu erscheinen: Nach Barck ,,impliziert [es] einen demokratischen egalitären Impuls, weil allen Menschen die prinzipielle Fähigkeit zur Poesie zuerkannt wird ${ }^{14}$ “" Gegen mögliche Vorwürfe einer autonomen, künstlichen Kunst wird die automatische Schreibweise als demokratisches Mittel interpretiert.

Die Druckgenehmigungsakten weisen ebenfalls auf die Argumente hin, die die Veröffentlichung im Kontext der DDR stützen konnten. Das unabhängige Gutachten wurde von Manfred Naumann, einem anerkannten Romanisten, verfasst und besteht aus fünf Seiten. Man kann

10. - Vgl. D. Risterucci-Roudnicky, France-RDA, a.a.O., S. 388.

11. - K. Barck, Surrealismus in Paris, a.a.O., S. 717.

12. - Ebd., S. 723.

13. - Vgl. D. Risterucci-Roudnicky, ebd., S. 394.

14. - K. Barck, ebd., S. 738. 
im Text drei thematische Ebenen erkennen. Naumann skizziert eine kurze Definition des Surrealismus und die Entwicklung der Bewegung, um die Leser des Gutachtens, d.h. das Personal der Abteilung Literatur und Buchwesen im Ministerium für Kultur, mit dem Thema vertraut zu machen. Diese Darstellung wird von einer strategischen Rhetorik begleitet, die für eine Veröffentlichung plädiert. Diese Rhetorik entspricht den Erwartungen, die 1960 in den „Richtlinien für die Begutachtung“ formuliert wurden. Dieser Text legt das Druckgenehmigungsverfahren fest und bestimmt die „Kriterien für die inhaltliche und ideologische Beurteilung von Manuskripten", damit eine Literatur im Einklang mit dem sozialistischen Aufbau entwickelt werden kann ${ }^{15}$. Als letzter Bestandteil des Gutachtens kommentiert der Verfasser die Arbeit von Karlheinz Barck, indem er u.a. formelle Kritiken an der Gestaltung der Anthologie äußert.

Esist festzustellen, dass die Argumentation des Gutachtens oft mit dem Inhalt des Nachworts von Barck vergleichbar ist. Naumann weist darauf hin, dass sich die surrealistischen Künstler als eine gegen die kapitalistische Gesellschaft politisch orientierte Bewegung betrachtet hatten. Ganz im Vordergrund des Gutachtens steht also der Wunsch der Surrealisten nach einer „Revolutionierung der bürgerlichen Gesellschaft.“ Dann aber nimmt Manfred Naumann Abstand zum Surrealismus. Er äußert sich kritisch, indem er auf das Scheitern der Bewegung hinweist: „An diesen Programmen war vieles Illusion.“ Diese kritische Einstellung ergibt sich aus „einem gewonnenen geschichtlichen Abstand“. Dies schließt an die Annahme von Barck an, dass „,der Surrealismus [...] Geschichte“ sei ${ }^{16}$. Diese Distanz ermöglicht eine dialektische Auseinandersetzung mit dieser künstlerischen Bewegung, anstatt sie wie bisher einfach abzulehnen. Dadurch wird eine Rechtfertigung der Rezeption surrealistischer Literatur möglich, wie man es in folgendem Zitat lesen kann: „,Die sozialistische Gesellschaft in ihrem jetzigen Entwicklungsstand kann sich nicht nur ein souveränes Verhältnis zum Surrealismus leisten, sie sollte sich auch ein solches zueigen machen 17 ." Erstens wird hier die DDR als reif genug betrachtet, um eine solche Literatur zu rezipieren. Zweitens wird das Argument verwendet, dass sich die DDR bisher unerwünschte Formen von Literatur aneignen und eine eigene Interpretation vorschlagen muss, um nicht von den westlichen Ländern überholt zu

15. - Vgl. Johanna Marschall-Reiser, Einleitung über die Druckgenehmigungsakte, im Online-Findbuch des Bundesarchivs, Ministerium für Kultur (DR 1), Teil 3: Hauptverwaltung Verlage und Buchhandel, Druckgenehmigungsvorgänge, S. 20. URL: http://www.argus.bstu.bundesarchiv.de/dr1_druck/xml/inhalt/dao/dr1druck_einleitung. pdf $[07 / 10 / 2016]$.

16. - K. Barck, Surrealismus in Paris, a.a.O., S. 717.

17. - Hervorhebung im Text. 
werden. Insgesamt schreibt Naumann eine sehr positive Einschätzung des Nachworts von Karlheinz Barck. Für ihn ist es ein Nachwort, das den Beitrag des Surrealismus zur Dichtung des 20. Jahrhunderts unterstreicht, wobei der Gutachter auch den distanzierten Umgang hervorhebt, mit dem Barck heikle Fragen behandelt. 